Methods We undertook a mixed survey with both quantitative and qualitative sections. This was analysed using a narrative approach.

Results All respondents feel the AIMP represents an improvement in the care offered to acutely ill patients in the hospice setting.

The Majority (60\%) felt the AIMP is acceptable in the hospice setting and (40\%) felt it is sometimes acceptable. No staff felt it was inappropriate in a hospice setting.

Additional comments include:

- Concerns about stopping treatment when a patient is felt to be clearly in the last days of life;

- Concerns about documenting individualised goals or triggers for medical review, to avoid frequent observations not leading to changes in treatment;

- A need for improved recording of urine output;

- A desire for more training in acute illness management.

Conclusions The AIMP acceptable in a hospice setting. Future work will include incorporating NEWS2 guidance, and an ongoing quality improvement programme to look at areas where it could be used more effectively. This will allow us to determine the efficacy of the AIMP along with ensuring staff feel empowered in the process of its introduction.

\section{ULTRASOUND GUIDED PARACENTESIS IN A HOSPICE SETTING: A CASE SERIES}

Andrew Tinker, Lynne Russon, Christopher Kane. Sue Ryder Wheatfields Hospice, Leeds

\subsection{6/bmispcare-2019-ASP.125}

Background Ultrasound guided paracentesis is a safe and effective methods of managing ascites caused by malignancy or cirrhosis. It has traditionally been performed in hospital and often requiring admission for one or more nights. Following training in ultrasound and gaining experience in ultrasound guided paracentesis at our local hospital trust, we evaluated the introduction of a hospice based paracentesis day-case programme.

Methods We undertook a prospective evaluation of the service over 3 years in specialist palliative care patients referred for assessment of possible ascites and where indicated draining ascites in a hospice setting, including patient feedback collected approximately a week after the procedure.

Results 85 assessments were performed for possible ascites (58\% non-hepatocellular cancer, 31\% cirrhosis \pm malignancy, $1 \%$ other), in 58 different patients. 45 patients were assessed as having paracentesis suitable for drainage in the hospice setting. This led to 36 successful ultrasound guided paracentesis procedures performed in the hospice inpatient unit $(9$ patients did not have paracentesis as not very symptomatic and/or too unwell). 21 were performed on a day case basis, 11 as part of a longer admission for symptom control or end of life care, and only 4 (16\% of admissions for paracentesis) requiring an overnight stay for the procedure. There were no complications

$64 \%$ of patients completed feedback a week later. 23/23 $(100 \%)$ patients would be happy to have the procedure repeated in the hospice setting if required. 16/23 (70\%) said paracentesis greatly helped symptoms, and 6/23 moderately $(26 \%)$
Conclusions It is practical and useful to include ultrasound in assessment of possible ascites in a hospice setting, and to use ultrasound guided paracentesis for day case, and existing inpatients in a hospice setting. This leads to reduced hospital attendance/admission, and it is an acceptable alternative to patients.

\section{INDWELLING PERITONEAL CATHETER INSERTION FOR MALIGNANT ASCITIES; SERVICE EVALUATION AND GUIDELINE PRODUCTION}

Leonie Armstrong, Felicity Dewhurst, Katie Frew, Robert Johnston, Sarah Robinson, Dawn Elliot, Avinash Aujayeb. Northumbria Health Care NHS Foundation Trust

\subsection{6/bmispcare-2019-ASP.126}

Background Conventional management of malignant ascites is recurrent inpatient paracenteses. Patients have high associated morbidity. Alternative management is insertion of an indwelling peritoneal catheter (IPC) supported by NICE, reported to have infrequent complications and associated with estimated cost saving of $£ 1051$ per patient. Our trust has an established indwelling pleural catheter service. In order to provide symptomatic benefit pleural and surgical consultants with ultrasound training started inserting IPCs in operating theatre under strict asepsis. The palliative care team is crucial to patient selection and follow up.

Methods We performed a retrospective trust-wide service evaluation followed by dissemination of results and clinical guideline production. A prospective service evaluation is ongoing. Results From January 2016 to April 2018, 11 patients had an IPC inserted. Median age was 67. Diagnoses were pancreatic cancer (3), mesothelioma (2), breast cancer (2), colorectal cancer (1), gastric cancer (1) and oesophageal cancer (1). Mean paracenteses prior to IPC was 3.45 and mean time from initial drainage to IPC and from IPC insertion to death was 53 and 55 days respectively. 1 complication (migration of the IPC precluding drainage) arose. Following the implementation of local guidelines, 8 IPCs were inserted insertions between April and September 2018 with no complications. Median age and diagnoses were similar. However mean paracenteses prior to IPC was 1.25 and mean time from initial drainage to IPC and from IPC insertion to death was 19 and 12 days respectively. In all cases IPC insertion was associated with significant symptomatic relief.

Conclusions IPC insertion for malignant ascites is a feasible, safe and effective method of management of malignant ascites. A proactive service reduces patient's hospital visits and procedure rate significantly. Formal guidelines meant that IPCs were inserted sooner in patients who previously would have likely died with significantly symptomatic untreated ascites.

\section{MODIFIED EARLY WARNING SCORE (EWS) TO AID IDENTIFICATION OF THE DETERIORATING PATIENT AT ST GEMMA'S HOSPICE}

Emily Frinton, Catherine Malia, Rebecca Owen, Hannah Zacharias. St Gemma's Hospice

\subsection{6/bmjspcare-2019-ASP.127}

Background On admission to St Gemma's Hospice (SGH), patient escalation status is determined, guiding decisions when acute deterioration occurs. In August 2018, a modified early 
warning score (EWS) was implemented to aid identification of deterioration in those appropriate for medical intervention. Similar hospice protocols were reviewed and adapted. Admission baseline EWS and planned observation frequency is recorded, with emphasis on deviation from baseline triggering medical assessment.

Methods Retrospective analysis of patient notes admitted to SGH during August $2018 \quad(n=39)$. Parameters recorded included: escalation status, planned observation frequency and adherence, baseline admission EWS documentation, EWS deviations prompting medical assessment and any resulting changes to patient management. The audit will continue incorporating September and October.

Results 23 patients (59\%) were for best supportive care only. Of the remainder $(n=16), 89 \%$ had baseline admission EWS documented. Observations were recorded as required in $63 \%$ cases $(n=10)$ and once daily in $37 \%$ $(n=6)$. All were compliant to planned frequency. EWS deviation prompted medical assessment on seven occasions. Of these, six EWS recordings were triggered by concern from nursing staff or patient. Management was changed on three occasions, with initiation of intravenous antibiotics, fluids or supplemental oxygen. Minor deviation from baseline EWS of up to two points did not automatically trigger medical assessment; this was at the discretion of nursing staff.

Conclusions Modified EWS may benefit the subset of palliative care patients suitable for life prolonging measures by allowing earlier identification of acute deterioration. Recording observations on an as required basis seems sufficient to detect clinical deterioration. Management was changed in the minority of cases, although these interventions were significant. Clear documentation of escalation status to avoid inappropriate observation monitoring is essential.

\section{ANTIMICROBIAL STEWARDSHIP IN A SPECIALIST PALLIATIVE CARE UNIT}

Jeanna Strutinsky-Mason, David Wenzel, Rebecca Payne, Katerina Georgiade, Barbara Powell. LOROS (Leicestershire Organisation for the Relief of Suffering)

\subsection{6/bmjspcare-2019-ASP.128}

Background Competency in Antimicrobial Stewardship relies on 'documentation in the prescription chart and/or in patients' clinical records regarding clinical indication, route, dose, duration and review date of antimicrobials'. This project evaluates compliance within a Specialist Palliative Care (SPC) Unit. With the value of early Palliative Medicine recognised during Oncology treatments and increasing numbers of patients with non-malignant diseases, SPC Units regularly see patients where proactive management of infections is indicated.

Methods Information about antibiotic use in the SPC inpatient-unit was collected on a standardised proforma over one month. Antibiotic use was identified through searches of the electronic prescribing system and electronic notes. We defined an antibiotic episode as 'antibiotic use separated from other antibiotic use by time regardless of the source being treated'. Antibiotics changed due to escalation/de-escalation/culture results would count as one antibiotic episode.

Results There were 28 antibiotic episodes out of 61 admissions. 22 patients had antibiotics (36\%). 17/22 patients had one antibiotic episode $(77.3 \%) ; 4 / 22$ had 2 antibiotic episodes (18.2\%); $1 / 22$ had 3 antibiotic episodes (4.5\%). Fourteen antibiotic episodes were commenced prior to admission and fourteen were commenced by hospice doctors. 27/28 episodes documented clinical indications, mostly within electronic notes rather than the prescribing system. 5/5 intravenous and $3 / 9$ oral antibiotic prescriptions commenced during admission were reviewed within 48-72 hours.

None of the oral antibiotics commenced prior to admission had documented reviews. Two antibiotic courses were commenced for symptom control (pain from cellulitis; delirium) rather than potential life-prolongation.

Conclusions Antimicrobial Stewardship is important. Strategies to improve concordance are under development, including auto-populating electronic notes/the prescribing system to review all antibiotics at $48-72$ hours. The next evaluation of practice should also capture compliance with local prescribing guidance, culture results and the intention of treatment.

\section{AUDIT OF EFFECTIVENESS OF IN-PATIENT CLINICAL MANAGEMENT ESCALATION PLAN}

Cathrine Vincent, Peter Day, Alpna Chauhan. John Eastwood Hospice

\subsection{6/bmjspcare-2019-ASP.129}

Background In 2017, a formal escalation planning process for the management of patients at a hospice inpatient unit was initiated in response to the inappropriate transfer of a patient to the acute setting out-of-hours. This initiative involves formation of an escalation plan (EP) in advance consisting of one of three categories; supportive hospice care, hospice escalation or hospital escalation.

Aims and objectives This study aims to answer the question 'Does advanced escalation planning and documentation improve patient care?'

Methods Data was collected retrospectively of 50 cases from the in-patient unit 1 year after the EP plan process was commenced. A staff questionnaire was also included. The notes were reviewed for details of any treatment escalations that occurred or were considered and this was compared to their escalation plan at the time.

Results The process is being used effectively with 94\% of patient's having an EP documented. There were 45 escalation events, mostly for symptom control, and nearly half the patients had at least 1 event during their admission which reflects the current level of intervention in palliative care. In 36\% the acute hospital was involved for symptom control and diagnostic investigations. 4\% were appropriately transferred from the hospice to the hospital for inpatient care. Escalation events matched the EP in $88 \%$ and where they didn't this involved patient choice or a rapid change in the patient's condition. The staff who engaged with the staff questionnaire reported finding the EP helpful.

Conclusion This audit shows overall positive results of engagement and clinical outcomes of this new initiative. It shows plans are formed in advance, they are documented, and clinical judgment is being used in individual situations to ensure appropriate clinical treatments are offered and outcomes are of benefit to the patient. 